\title{
Review of: "Traditional Serrated Adenoma of the Gallbladder, a Case Report"
}

\author{
Lovenish Bains
}

Potential competing interests: The author(s) declared that no potential competing interests exist.

Micsik and colleagues report of Traditional Serrated Adenoma of the Gallbladder is an excellent submission, which may suggest the role of adenoma-carcinoma sequence in Gall Bladder cancer and open door to further insights. The case report has been rightly structured and has adequate flow of the manuscript. The concept of extra-colonic traditional serrated adenoma and its role remains investigational. Further cases will throw more light on the subject.

As it suggested a polypoid lesion; the size of the lesion on ultrasound could have been mentioned. The location of the polyp (esp. if the lesion was on the posterior wall or towards liver) may had defined role for CT scan. As neoplastic process cannot be determined on gross; the standard teaching should be that all such suspicious gall bladder should be removed in a pouch/bag without contaminating the port site. Congratulations to the Authors. 